\title{
Experimental assessment of anomalous low-frequency noise increase at the onset of Gunn oscillations in InGaAs planar diodes
}

\author{
Ó. García-Pérez, ${ }^{1, a)}$ Y. Alimi, ${ }^{2}$ A. Song, ${ }^{2}$ I. Íñiguez-de-la-Torre, ${ }^{1}$ S. Pérez, ${ }^{1}$ J. Mateos, ${ }^{1}$ \\ and T. González ${ }^{1}$ \\ ${ }^{1}$ Dpto. Física Aplicada, Universidad de Salamanca, 37008 Salamanca, Spain \\ ${ }^{2}$ School of Electrical and Electronic Engineering, University of Manchester, Manchester M13 9PL, \\ United Kingdom
}

(Received 2 July 2014; accepted 7 September 2014; published online 15 September 2014)

\begin{abstract}
In this work, the presence of anomalous low-frequency fluctuations during the initiation of higher frequency oscillations in InGaAs-based Gunn planar diodes has been evidenced and investigated. Accurate measurements showing the evolution of the power spectral density of the device with respect to the applied voltage have been carried out. Such spectra have been obtained in the wide frequency range between $10 \mathrm{MHz}$ and $43.5 \mathrm{GHz}$, simultaneously covering both the low-frequency noise and the fundamental oscillation peak at some tens of $\mathrm{GHz}$. This provides valuable information to better understand how these fluctuations appear and how these are distributed in frequency. For much higher frequency operation, such understanding can be utilized as a simple tool to predict the presence of Gunn oscillations without requiring a direct detection. (C) 2014 AIP Publishing LLC. [http://dx.doi.org/10.1063/1.4896050]
\end{abstract}

During the last decades, Gunn diodes have been widely used for the implementation of microwave oscillators. More recently, the improvement of the fabrication techniques, as well as the research on new materials and structures, have made possible the fabrication of Gunn diodes working up to several hundreds of $\mathrm{GHz}^{1-3}$ and the design of structures prospectively reaching $\mathrm{THz}$ frequencies. ${ }^{4}$ The oscillatory regime in such devices is usually considered as directly activated above a given threshold voltage. However, several studies have shown that the transition between a static state and the generation of a purely periodic signal is not so immediate, passing through a range of bias voltages for which unusual fluctuations appear in the device. ${ }^{5-9}$ On the one hand, some noise is formed around the characteristic frequency of the oscillator that, for a higher voltage, usually converges into a single coherent peak. Besides the previous wellknown effect, Refs. 5-9 also demonstrated the presence of an anomalous low-frequency noise (ALFN) preceding the high-frequency oscillations, which vanishes when the fundamental peak is well established. Accordingly, Matsuno ${ }^{5}$ reported strong current fluctuations at $100 \mathrm{kHz}$ in a very limited voltage range near the onset of the Gunn instability in a GaAs sample. Similarly, Kabashima et al. ${ }^{6}$ observed a current increase of several orders of magnitude at $50 \mathrm{kHz}$ for voltages just below the generation of a pure oscillation in a $\mathrm{X}$-band GaAs Gunn diode. In the latter study, such increase was attributed to the coexistence of both oscillatory and nonoscillatory states alternatively occurring with a random period, for certain range of voltages. The cathode contact characteristics can also be the origin of an increase of noise by leading to stationary field configurations in which local regions of negative differential mobility exist. ${ }^{10}$ More recently, the study of this ALFN is gaining a lot of interest for the characterization of new $\mathrm{mm}$-wave and $\mathrm{THz}$ Gunn

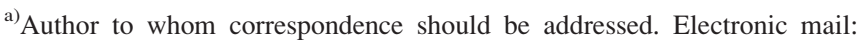
ogarcia@usal.es
}

sources. Following this line, Starikov et al., ${ }^{7}$ Shiktorov et al. ${ }^{8}$ and Íñiguez-de-la-Torre et al. ${ }^{9}$ used Monte Carlo particle methods to simulate the spectral density evolution in $\mathrm{InN}$, InGaAs, InP, and $\mathrm{GaN}$ devices working above $100 \mathrm{GHz}$, also predicting a noticeable enhancement of lowfrequency noise at the onset of the oscillations.

This manuscript presents wide-frequency spectral power measurements from InGaAs-channel Gunn diodes oscillating at some tens of GHz. In this context, the aim of the present letter is double. On the one hand, first purpose is to provide a complete field of view of the simultaneous evolution of both the low-frequency noise and the main oscillation peak in a Gunn diode for different supply voltages. It extends the experimental results presented in the literature that were restricted to very limited frequency ranges. ${ }^{5,6}$ Moreover, these results allow us to clearly identify the ALFN, analyze its dependence with frequency, and qualitatively correlate its appearance with the high-frequency oscillation. On the other hand, the second goal is more specific, and deals with the InGaAs-based planar diodes, and its demonstrated suitability to operate at mm-wave $\mathrm{e}^{1,2}$ and $\mathrm{THz}^{3,4}$ frequencies. The power measurement at hundreds of $\mathrm{GHz}$, typically made by means of sub-harmonic mixers or power meters, ${ }^{2}$ may be strongly limited by the inherent high conversion losses or reduced sensitivity of the previous instruments at so high frequencies. For $\mathrm{THz}$ devices, the scenario becomes even more challenging, usually requiring free space setups and relatively high power levels to detect the presence of oscillations in the source. ${ }^{11}$ Therefore, the knowledge on the ALFN mechanisms and its corresponding identification is proposed in this case as an alternative indirect method to determine the activation of higher frequency oscillations in devices based on similar InGaAs epitaxial structures properly adapted for (sub-) THz generation.

The diodes characterized in this work are based on an epitaxial layer structure consisting of an un-doped $\operatorname{In}_{0.23} \mathrm{Ga}_{0.77} \mathrm{As}$ channel layer placed between two $\mathrm{Al}_{0.23} \mathrm{Ga}_{0.77}$ As layers with 
double $\delta$-doped layers. The sheet density of each $\delta$-doped layer is $8 \times 10^{11} \mathrm{~cm}^{-2}$. Multiple $\mathrm{GaAs} / \mathrm{InGaAs}$ layers were grown over the active layers to improve the formation of good anode and cathode ohmic contacts. More details about the epitaxial structure are described in Ref. 1. After etching a mesa and removing the GaAs/InGaAs cap layers in the active region, the anode and cathode regions and a $50 \Omega$ coplanar waveguide (CPW) transmission line were patterned by thermal evaporation of $\mathrm{Pd} / \mathrm{Ge} / \mathrm{Au} / \mathrm{Pd} / \mathrm{Au}$ metals. The measured contact resistance is $0.08 \Omega \mathrm{mm}$. Due to their planar nature, the frequency of oscillation of this kind of diodes is directly determined by the anode-to-cathode separation. ${ }^{1}$ Actually, for our study, several Gunn devices with $60 \mu \mathrm{m}$ width and lengths around $3.0 \mu \mathrm{m}$ have been fabricated and characterized, obtaining equivalent conclusions in all the cases. First of all, the DC current-voltage (I-V) characteristics of a diode with $3.1 \mu \mathrm{m}$ length was measured with a semiconductor analyzer, model 4200-SCS from Keithley Instruments, whose curve is plotted in Fig. 1. As it can be seen in the figure, and in agreement with the results in Ref. 1, this kind of devices do not show prominent negative differential resistance in the I-V plot even though the Gunn effect is clearly activated in such voltage range according to the measured spectra and reflection coefficients. This indicates that the negative differential resistance effect is not present at low frequency and therefore the instabilities could only appear at high frequencies.

For the noise power characterization of the diodes, a vector network analyzer (VNA) with noise measurement capabilities, model PNA-X N5244A with Option 029 from Agilent Technologies, has been utilized for the frequency range between $10 \mathrm{MHz}$ and $43.5 \mathrm{GHz}$. This equipment allows, with a single connection to the 1-port device, the acquisition of both the reflection coefficient and the output noise power density in the frequency domain. Actually, a dedicated low-noise receiver is used for the noise measurement, whereas a conventional receiver is used for the scattering parameters in the same port. This in turn requires an additional calibration step of the noise receiver using a noise source or a power meter, but severely improves the accuracy and sensitivity of the instrument compared with a conventional spectrum analyzer. More information about the

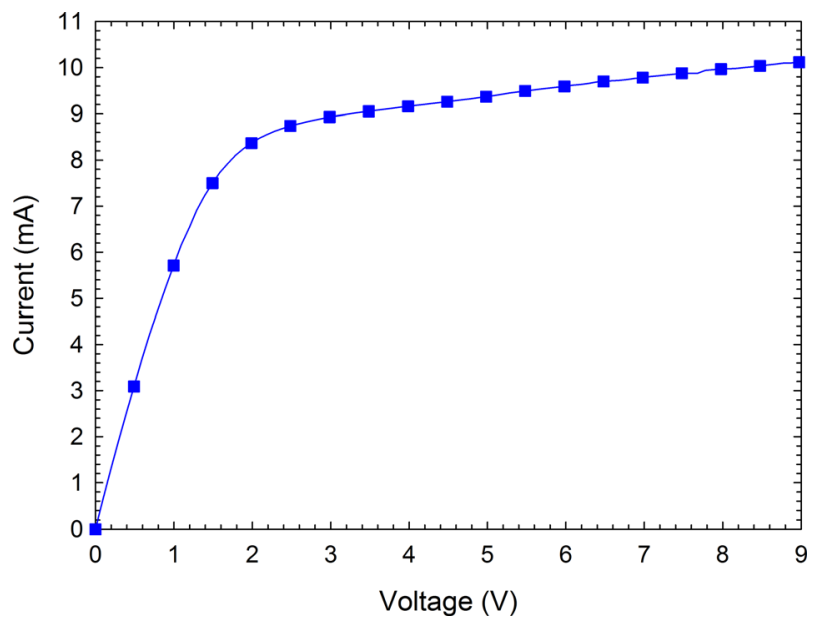

FIG. 1. Measured current-voltage characteristics of a $3.1 \mu \mathrm{m}$ length Gunn diode. performance and operation principles of the PNA-X can be obtained from the application note in Ref. 12. A sketch of the measurement setup is depicted in Fig. 2. After an onwafer calibration, the reference plane of the measurement is placed at the end of the CPW transmission line that gives access to the diode, which means that the losses of the cable and the probe are automatically eliminated in the experimental results shown below. The bias voltage is applied to the device under test by using the internal bias-tee of the VNA.

In order to illustrate the noise evolution of these diodes with respect to the bias voltage, the measured power spectra for a device of $3.1 \mu \mathrm{m}$ length are depicted in Fig. 3. When zero voltage is applied to the device, the spectral response is flat, and slightly lower than $-174 \mathrm{dBm} / \mathrm{Hz}$. This noise value corresponds to the available power density in form of thermal noise of a passive device at room temperature, ${ }^{13}$ in this case weighted by the impedance mismatching of the diode in the reference plane, i.e.,

$$
\begin{aligned}
\left.S^{n}(f)\right|_{\mathrm{V}=0 \mathrm{~V}} & =10 \log \left(k T_{0}\left(1-\left|s_{11}(f)\right|^{2}\right)\right) \\
& =-174 \mathrm{dBm} / \mathrm{Hz}+10 \log \left(1-\left|s_{11}(f)\right|^{2}\right),
\end{aligned}
$$

where $S^{n}$ denotes the spectral noise power density, $f$ is the frequency, $k$ is the Boltzmann constant, $T_{O}=290 \mathrm{~K}$ refers to the room temperature, and $s_{11}$ is the reflection coefficient of the device-under-test (in this case $\left|s_{11}(f)\right| \approx 0.48$ according to the measurements). Also, it should be noticed that some undesired radiofrequency interferences appear at around $100 \mathrm{MHz}$ and $2.4 \mathrm{GHz}$, which correspond to the well localized communication services of FM radio and $\mathrm{Wi}-\mathrm{Fi}$, respectively. Nevertheless, their effect can be just ignored due to their narrowband characteristics. When the DC current begins to flow through the diode, e.g., at $V=1.0 \mathrm{~V}$, flicker noise immediately arises at low frequencies with its characteristic $1 / f$ dependence. Up to $V=5.0 \mathrm{~V}$, both flicker noise and thermal noise increases with the applied DC voltage. At $V=5.5 \mathrm{~V}$, two effects can be observed in the graph. First, high-frequency oscillations begin to be formed above $40 \mathrm{GHz}$, and second, a small hump appears between $100 \mathrm{MHz}$ and $10 \mathrm{GHz}$. When increasing the voltage up to $V=7.0 \mathrm{~V}$ and $V=8.0 \mathrm{~V}$, the main oscillation grows up in magnitude and shifts down in frequency to around $30 \mathrm{GHz}$. Furthermore, the previous hump significantly increases between $100 \mathrm{MHz}$ and $1 \mathrm{GHz}$, evidencing the presence of the

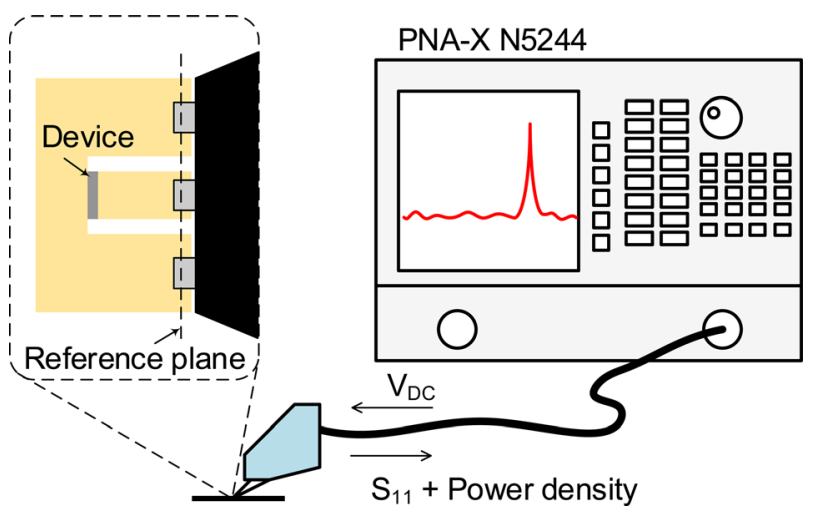

FIG. 2. Experimental setup for the measurement of the reflection coefficient and the power spectral density. 


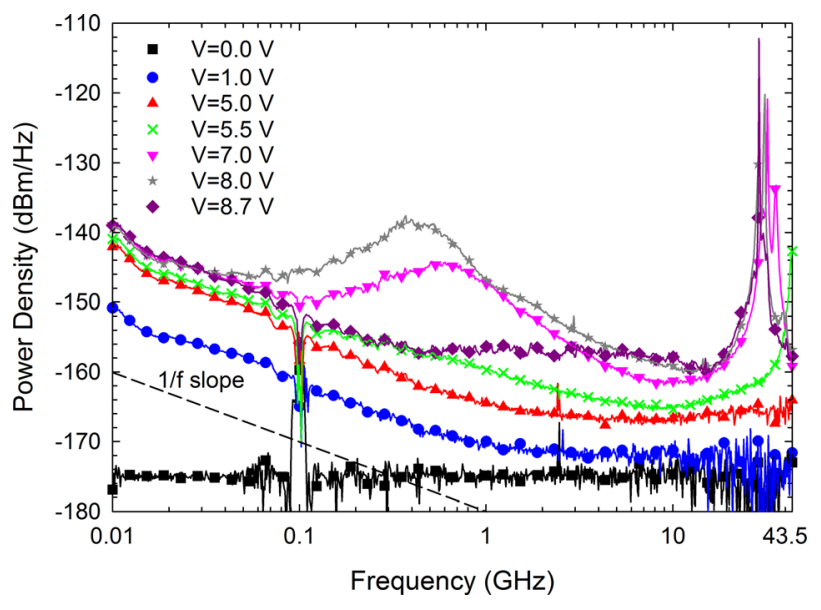

FIG. 3. Measured power density spectrum of a $3.1 \mu \mathrm{m}$ length Gunn diode for different DC voltages.

ALFN. It should be noted that this type of noise cannot be attributed to neither flicker nor thermal noise, whose effects can still be extrapolated from the curves and their frequency dependence is totally different to the observed response. When the voltage reaches $V=8.7 \mathrm{~V}$, the main oscillation forms a sharp peak at about $29 \mathrm{GHz}$, and the preceding lowfrequency fluctuations are drastically reduced. At this point, it should be remarked the tremendous similarity between the described experimental results and the simulated spectra predicted from the Monte Carlo simulation in Ref. 8 for a GaAs Gunn device. Another point of interest that can be extracted from the results is that, when the ALFN is more prominent, the power seems to be concentrated around certain defined frequency, which roughly corresponds to $400 \mathrm{MHz}$. An equivalent behavior was observed for other devices of different lengths as far as they oscillate, for which the ALFN also appeared in the same frequency range. As suggested in Ref. 6, this behavior could be originated by initially intermittent oscillations randomly built up and characterized by a given delay time, leading to an anomalous noise increase. This hypothesis seems to reasonably explain the origin and nature of the ALFN in the present results, since such a characteristic delay time would correspond in this case with the frequency range in which the ALFN power concentrates. The interpretation of the phenomenon in Ref. 8 is quite similar: at the onset of the instabilities uncorrelated current pulses, whose power concentrates at low frequencies, spontaneously appear; then, for higher bias, the pulse sequence becomes coherent and forms a continuous periodic signal with all the power concentrated in resonant peaks at high frequencies.

Other effects such as self-mixing were discarded as origin of the ALFN, since the separation between the highfrequency peaks is significantly larger (several $\mathrm{GHz}$ ) than the frequency around which it concentrates. On the other hand, inhomogeneities at the cathode contact leading to saturated I-V curves, ${ }^{14-16}$ like those exhibited by our devices, could also be at the origin of a noise enhancement in diodes not exhibiting oscillations unless connected to a resonant circuit. ${ }^{10}$ However, this does not seem to be the origin of the effect we observe, since: (i) simulations of diodes with ideal contacts show a very similar noise enhancement, ${ }^{8}$ (ii)

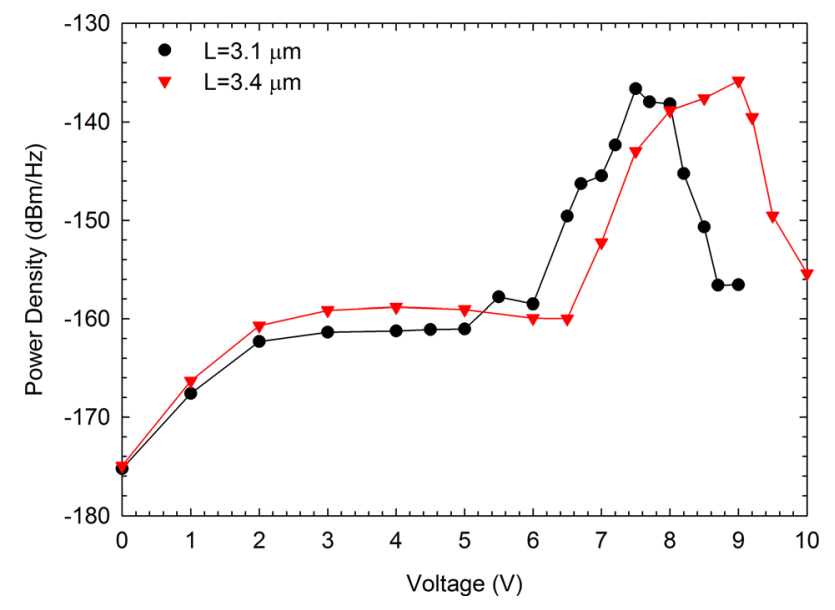

FIG. 4. Measured noise power density at $400 \mathrm{MHz}$ of two Gunn diodes of $3.1 \mu \mathrm{m}$ and $3.4 \mu \mathrm{m}$ lengths.

measurements in diodes exhibiting a standard Gunn-like I-V curve (with negative differential resistance) show also indications of a noise increase at low frequencies. ${ }^{6}$ Moreover, in both cases, the noise increase is strictly linked to the onset of the oscillations, vanishing once these are consolidated.

In order to clearly appreciate the growth and subsequent fading of the low-frequency noise, the power density with respect to the voltage at $400 \mathrm{MHz}$, where the ALFN presents greater excursion in magnitude, is represented in Fig. 4. In order to show the repeatability of the results, the curve for a second sample of $3.4 \mu \mathrm{m}$ length is also plotted for comparison. Three regions are clearly observed from the curves. In the first range, for voltages below 6.0 and $6.5 \mathrm{~V}$ for the devices of 3.1 and $3.4 \mu \mathrm{m}$, respectively, the diode does not oscillate and only $1 / f$ noise appears. Therefore, such noise describes a curve essentially proportional to the bias current ${ }^{17}$ in the mentioned range. In the subsequent range, up to 8.5 and $9.5 \mathrm{~V}$, respectively, the diode begins to be unstable and it can be clearly seen in the curves how the ALFN dominates and becomes more than one order of magnitude higher than the flicker noise. In contrast, for upper voltages the diode provides a purer oscillation mode and the ALFN level clearly decays.

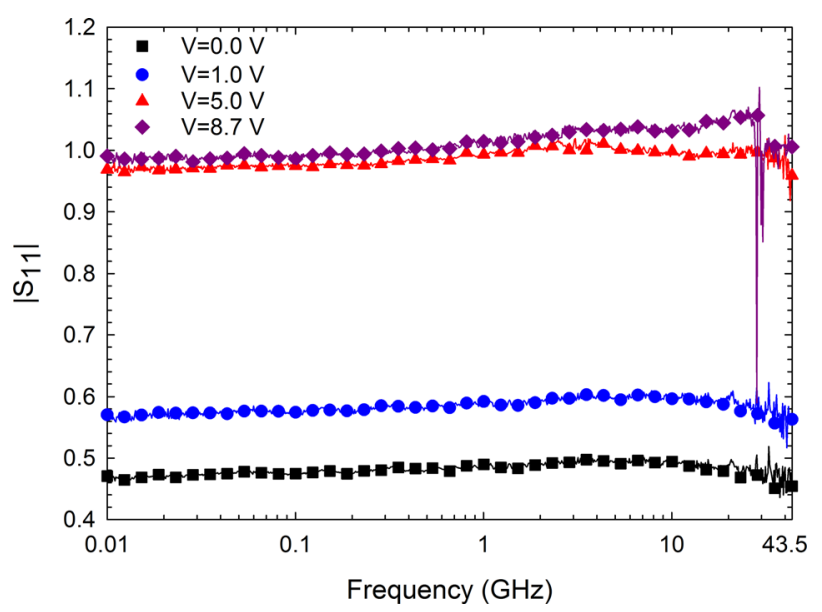

FIG. 5. Measured reflection coefficient of a $3.1 \mu \mathrm{m}$ length Gunn diode for different DC voltages. 
Finally, the last presented result corresponds with the evolution of the reflection coefficient with respect to the voltage for the device of $3.1 \mu \mathrm{m}$ length, shown in Fig. 5. From the plot, it can be seen how the reflection parameter $s_{11}$ increases with voltage up to the threshold of the instable zone (i.e., $\left|s_{11}\right|=1$ ). When the oscillation is evident, e.g., at $8.7 \mathrm{~V}$ in this case, $s_{11}$ becomes higher than one in magnitude at high frequencies, and some resonant peaks appear at the frequency of the main oscillation due to the excess of power captured by the receiver at this point.

In summary, the evolution of the spectral power density of InGaAs planar Gunn diodes has been thoroughly characterized, allowing us to confirm and analyze the presence of low-frequency noise during the formation of higher frequency oscillations. At frequencies well below the main oscillation, such noise significantly increases for certain range of voltages. Furthermore, at some point, its power seems to concentrate around a characteristic frequency. Lastly, when the oscillation becomes spectrally purer at higher voltages, the referred noise disappears. Understanding the described mechanisms can be used as indicator to predict very high frequency Gunn oscillations in scenarios in which the lack of adequate instrumentation and/or the constraints due to losses, noise or low-power levels hinder a proper detection. This could be the case of GaN diodes, ${ }^{9}$ where difficulties are met to experimentally evidence the presence of oscillations. $^{18}$

This work has been partially supported by the Spanish MINECO through project TEC2013-41640-R and by the Consejería de Educación de la JCyL through project
SA052U13. Ó. García-Pérez wants to thank the European Social Fund (ESF) for financing his postdoctoral contract.

${ }^{1}$ C. Li, A. Khalid, S. H. Paluchowski-Caldwell, M. C. Holland, G. M. Dunn, I. G. Thayne, and D. R. S. Cumming, Solid State Electron. 64, 67 (2011).

${ }^{2}$ A. Khalid, C. Li, V. Papageorgiou, G. M. Dunn, M. J. Steer, I. G. Thayne, M. Kuball, C. H. Oxley, M. Montes Bajo, A. Stephen, J. Glover, and D. R. S. Cumming, IEEE Electron Device Lett. 34, 39 (2013).

${ }^{3}$ A. Khalid, G. M. Dunn, R. F. Macpherson, S. Thoms, D. Macintyre, C. Li, M. J. Steer, V. Papageorgiou, I. G. Thayne, M. Kuball, C. H. Oxley, M. Montes Bajo, A. Stephen, J. Glover, and D. R. S. Cumming, J. Appl. Phys. 115, 114502 (2014).

${ }^{4}$ S. Pérez, T. González, D. Pardo, and J. Mateos, J. Appl. Phys. 103, 094516 (2008).

${ }^{5}$ K. Matsuno, Phys. Lett. A 31, 335 (1970).

${ }^{6}$ S. Kabashima, H. Yamazaki, and T. Kawakubo, J. Phys. Soc. Jpn. 40, 921 (1976).

${ }^{7}$ E. Starikov, P. Shiktorov, V. Gruzinskis, L. Reggiani, L. Varani, and J. C. Vaissiere, AIP Conf. Proc. 780, 791 (2005).

${ }^{8}$ P. Shiktorov, E. Starikov, V. Gruzinskis, L. Varani, and L. Reggiani, AIP Conf. Proc. 1129, 179 (2009).

${ }^{9}$ A. Íñiguez-de-la-Torre, I. Íñiguez-de-la-Torre, J. Mateos, and T. González, Appl. Phys. Lett. 99, 062109 (2011).

${ }^{10}$ M. P. Shaw, P. R. Solomon, and H. L. Grubin, IBM J. Res. Dev. 13, 587 (1969).

${ }^{11}$ A. Westlund, P. Sangaré, G. Ducournau, P. A. Nilsson, C. Gaquiere, L. Desplanque, X. Wallart, and J. Grahn, Appl. Phys. Lett. 103, 133504 (2013).

${ }^{12}$ Agilent Technologies Inc., Application Note 1408-20 (5990-5800EN), USA (2013).

${ }^{13}$ D. M. Pozar, Microwave Engineering (John Wiley and Sons, New York, 1998), p. 551.

${ }^{14}$ J. B. Gunn, IBM J. Res. Dev. 10, 300 (1966).

${ }^{15}$ M. Ohtomo, Jpn. J. Appl. Phys. 7, 1368 (1968).

${ }^{16}$ M. P. Shaw, P. R. Solomon, and H. L. Grubin, Appl. Phys. Lett. 17, 535 (1970).

${ }^{17}$ A. van der Ziel, Proc. of the IEEE 58, 1178 (1970).

${ }^{18}$ O. Yilmazoglu, K. Mutamba, D. Pavlidis, and T. Karaduman, IEEE Trans. Electron. Devices 55, 1563 (2008). 